\title{
Vascular Deformation Mapping (VDM) of thoracic aortic aneurysm: an application for color 3D printing in aortic disease
}

\author{
Nicholas S. Burris ${ }^{1}$, Benjamin A. Hoff ${ }^{1,2}$, Brian D. Ross ${ }^{1,2,3}$ \\ ${ }^{1}$ Department of Radiology, ${ }^{2}$ Center for Molecular Imaging (CMI), ${ }^{3}$ Department of Biologic Chemistry, University of Michigan, Ann Arbor, MI, USA \\ Correspondence to: Nicholas S. Burris, MD. Department of Radiology, University of Michigan, 1500 E. Medical Center Drive, TC B1-132, SPC-5030, \\ Ann Arbor, MI 48109-5030, USA. Email: nburris@med.umich.edu. \\ Provenance: This is an invited Editorial commissioned by Section Editor Busheng Zhang, MD, PhD (Department of Cardiac Surgery, Shanghai Chest \\ Hospital, Shanghai Jiao Tong University, Shanghai, China). \\ Comment on: Burris NS, Hoff BA, Kazerooni EA, et al. Vascular Deformation Mapping (VDM) of Thoracic Aortic Enlargement in Aneurysmal \\ Disease and Dissection. Tomography 2017;3:163-73.
}

Submitted Dec 04, 2018. Accepted for publication Dec 10, 2018.

doi: $10.21037 /$ atm.2018.12.16

View this article at: http://dx.doi.org/10.21037/atm.2018.12.16

$3 \mathrm{D}$ printing is a rapidly evolving technology at the forefront of biomedical innovation and cardiovascular 3D printing is one of the most common medical applications (1). Technical advances in biomedical $3 \mathrm{D}$ printing have been driven by improved printer technology and image segmentation tools, however, clinical and research applications of cardiovascular are still catching up to such rapid technical advances. While traditionally medical 3D printing has focused on the creation of anatomical models from imaging data [e.g., computed tomography (CT), magnetic resonance imaging (MRI)] using a single material, recent advances in multi-material printing technology have allowed for generation of $3 \mathrm{D}$ printed models with variable properties such as flexibility and color. Material flexibility has been shown to be a particularly appealing property for cardiovascular applications in the field of structural heart disease, specifically for procedural planning in transcatheter aortic valve replacement (TAVR) where various valve size and design characteristics can be studied within a flexible, patient-specific model of the aortic root to determine optimal device selection and deployment (2). Additionally, physiologic properties of the diseased valve in aortic stenosis (e.g., pressure gradient, flow acceleration) can be simulated through the use of $3 \mathrm{D}$ printed models with tissue-specific elastic properties (3). Recent advances in $3 \mathrm{D}$ printing techniques have also allowed for the creation of models containing a full range of color. Full spectrum color 3D printing applications may be particularly useful for biomedical applications. One potential use of color
$3 \mathrm{D}$ printing in biomedical $3 \mathrm{D}$ printing is to depict the relationship of anatomic and functional data using colorcoded physiological data, essentially creating a model that fuses "form" and "function".

Our group has recently developed a novel image processing technique, termed Vascular Deformation Mapping (VDM), that yields a 3D assessment of aortic growth in patients with thoracic aortic aneurysm (TAA) (4). In brief, the VDM analysis workflow involves aortic segmentation of two computed tomography angiography (CTA) examinations, followed by image registration and measurement of local aortic wall deformation (i.e., strain) between baseline and follow-up time points using a spatial Jacobian analysis, which is then normalized by the time interval to yield aortic growth rate. The $3 \mathrm{D}$ nature of VDM allows for assessment of aortic enlargement along the entire length and circumference of the vessel wall and allows for aortic growth rate measurements to be depicted as a "heat map" that is superimposed on the patient's aortic anatomy yielding a functionalized model. Given that the output of VDM analysis yields both anatomic and color-coded aortic growth data, the technique is well-suited for color $3 \mathrm{D}$ printing. As an example of the potential applicability of color 3D printing to VDM we present the case of a young patient with a rapidly expanding ascending aortic aneurysm who underwent CTA evaluation for the purposes of surgical planning (5). VDM analysis was performed as part of a retrospective institutional review board approved study. Results of VDM analysis were superimposed on a 


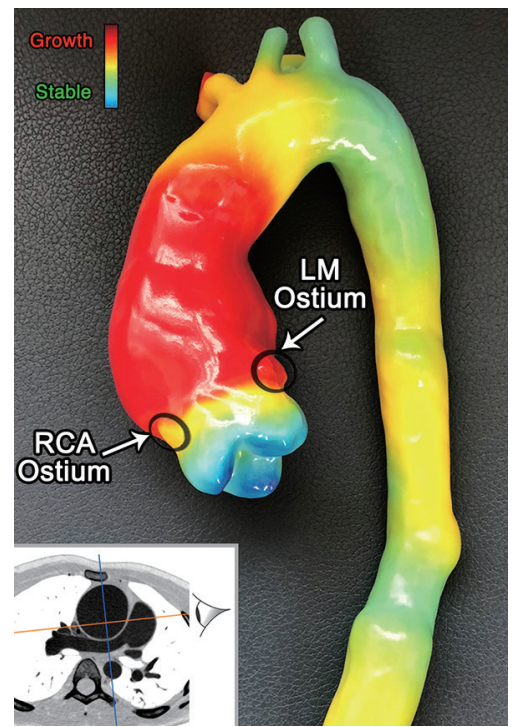

Figure 1 Photograph of a color 3D printed model of the VDM results shown in a left anterior oblique orientation, demonstrating heterogeneous growth in the thoracic aorta. The aorta model was printed using a digital CT image file using PolyJet 3D printing technique, which involves deposition of resin droplets that are cured through exposure to ultra violet light (J750 3D Printer, Stratasys, Ltd.). This process allowed for 3D printing resolution of 14 microns per layer and multi-material model composition in full color scale. Following printing of the aorta model, it was cleaned and clear coated to provide a glossy finish. Color 3D printed models could be valuable for both surgical planning and patient education purposes. VDM, Vascular Deformation Mapping; CT, computed tomography.

model of the patient's thoracic aorta and were printed on a color 3D printer using PolyJet method (J750 3D Printer, Stratasys, Ltd.) (Figure 1). This patient's VDM model clearly demonstrates high intensity growth (red) in the ascending aorta, with lower intensity gradations of growth involving the proximal portions of the aortic arch (yellow) and areas of stability (green) in the distal portions of the aortic arch. The color 3D printed model of this patient's thoracic aorta was reviewed with the cardiac surgeon who had repaired this patient's aneurysm and feedback was universally positive. Specifically, the surgeon noted that such models could help plan a surgical resection strategy that maximized removal of growing segments of aortic tissue, while preserving non-growing segments. In this case, the coronary arteries were noted by the surgeon to arise from areas of growing proximal ascending aorta, suggesting the need for a surgical approach that requires coronary artery manipulation, thus increasing the technical demands of the surgical repair. An added benefit of 3D printing of VDM results is that surgeons are able to handle the functionalized model of the aorta and understand how the patient's specific anatomical landmarks relate to the distribution of aortic growth considering that this relationship would have to be thoroughly understood by the surgeon to permit a selective aortic resection strategy in the operating room. Additionally, we believe that color 3D-printed models of VDM results could play an important role in pre-operative patient education. Many patients with aortic disease have a limited understanding of their disease process, creating barriers to optimal care. Additionally, patients are often asked to undertake aggressive pre-operative regimen (e.g., dietary or lifestyle medications) to minimize their operative risks and we believe that the availability of a color $3 \mathrm{D}$ printed VDM model would allow surgeons to not only more effectively explain the surgical repair strategy, but also to more clearly confer the gravity of the patient's aortic disease with the goal of increasing patient participation in decision making and engagement in their pre- and postoperative regimens.

Continued advancements in 3D printing technology have allowed for creation of increasingly sophisticated models composed of multiple materials with variable material stiffness and color among other properties. Beyond simply using color to differentiate certain anatomic structures (e.g., arteries $v s$. veins, left $v s$. right ventricle) we demonstrate that full spectrum color $3 \mathrm{D}$ printing could also be used to display the distribution of color-coded physiologic information on an anatomic model, adding a 4th dimension to a standard 3D model. In our specific application of color $3 \mathrm{D}$ printing in VDM, the functionalized model displays a patient specific aneurysm morphology with a color overlay of aortic growth measurements, although many other potentially useful physiologic parameters could be displayed in a similar manner. Examples of cardiovascular applications that could potentially benefit from color 3D printing with fused anatomic-physiologic data include atrial voltage/wall thickness mapping for atrial ablation planning, myocardial tissue characterization (e.g., T1, T2 and delayed enhancement mapping) prior to endomyocardial biopsy or ventricular ablation, and MRI-derived aortic wall shear stress mapping prior to aortic surgery (6-10).

In summary, we believe that color 3D printing has many potential clinical and research applications in cardiovascular disease and highlight VDM assessment of aortic growth in 
TAA as an example of just one new potential application. In future work we hope to further improve our models with the addition of flexible and hollow materials with anatomically correct wall thickness to better simulate realistic aortic properties. As full spectrum color 3D printers become more widely available and affordable we envision many applications in advance surgical/procedural planning, patient education and biomedical research that will advance the understanding and treatment of cardiovascular disease.

\section{Acknowledgements}

None.

\section{Footnote}

Conflicts of Interest: The authors have no conflicts of interest to declare.

\section{References}

1. Vukicevic M, Mosadegh B, Min JK, et al. Cardiac 3D Printing and its Future Directions. JACC Cardiovasc Imaging 2017;10:171-84.

2. Ripley B, Kelil T, Cheezum MK, et al. 3D printing based on cardiac $\mathrm{CT}$ assists anatomic visualization prior to transcatheter aortic valve replacement. J Cardiovasc Comput Tomogr 2016;10:28-36.

3. Maragiannis D, Jackson MS, Igo SR, et al. Replicating Patient-Specific Severe Aortic Valve Stenosis With

Cite this article as: Burris NS, Hoff BA, Ross BD. Vascular Deformation Mapping (VDM) of thoracic aortic aneurysm: an application for color 3D printing in aortic disease. Ann Transl Med 2018;6(Suppl 2):S123. doi: 10.21037/atm.2018.12.16
Functional 3D Modeling. Circ Cardiovasc Imaging 2015;8:e003626.

4. Burris NS, Hoff BA, Kazerooni EA, et al. Vascular Deformation Mapping (VDM) of Thoracic Aortic Enlargement in Aneurysmal Disease and Dissection. Tomography 2017;3:163-73.

5. Burris NS, Hoff BA, Patel HJ, et al. Three-Dimensional Growth Analysis of Thoracic Aortic Aneurysm With Vascular Deformation Mapping. Circ Cardiovasc Imaging 2018;11:e008045.

6. Bollache E, Fedak PW, van Ooij P, et al. Perioperative evaluation of regional aortic wall shear stress patterns in patients undergoing aortic valve and/or proximal thoracic aortic replacement. J Thorac Cardiovasc Surg 2018;155:2277-86.e2.

7. Ferreira VM, Piechnik SK, Robson MD, et al. Myocardial tissue characterization by magnetic resonance imaging: novel applications of T1 and T2 mapping. J Thorac Imaging 2014;29:147-54.

8. Bishop M, Rajani R, Plank G, et al. Three-dimensional atrial wall thickness maps to inform catheter ablation procedures for atrial fibrillation. Europace 2016;18:376-83.

9. Rolf S, Hindricks G, Sommer P, et al. Electroanatomical mapping of atrial fibrillation: Review of the current techniques and advances. J Atr Fibrillation 2014;7:1140.

10. Dickfeld T, Tian J, Ahmad G, et al. MRI-Guided ventricular tachycardia ablation: integration of late gadolinium-enhanced $3 \mathrm{D}$ scar in patients with implantable cardioverter-defibrillators. Circ Arrhythm Electrophysiol 2011;4:172-84. 